\title{
Towards omnidirectional, large scale, full polarization, and broadband practical invisibility cloaks: challenges and progress
}

\author{
Yihao Yang ${ }^{1,2}$, Baile Zhang ${ }^{3,4}$, Erping $\mathrm{Li}^{2,3,4}$, and Hongsheng Chen ${ }^{1,2, *}$ \\ 1 The Electromagnetics Academy at Zhejiang University, Zhejiang University, Hangzhou 310027, P.R. China \\ 2 Department of Information Science and Electronic Engineering, Zhejiang University, Hangzhou 310027, P.R. China \\ 3 Division of Physics and Applied Physics, School of Physical and Mathematical Sciences, Nanyang Technological University, \\ 637371 Singapore \\ 4 Centre for Disruptive Photonic Technologies, Nanyang Technological University, 637371 Singapore
}

Received 25 June 2014 / Accepted 21 December 2014

\begin{abstract}
Invisibility cloaks have experienced a tremendous development in the past few years, but the current technologies to convert the cloaks into practical applications are still facing numerous bottlenecks. In this paper, we provide the review of the challenges and recent progress in the invisibility cloaks from a practical perspective. In particular, the following key challenges such as non-extreme parameters, homogeneity, omnidirectivity, full polarization, large scale and broad band are addressed. We analyze the physical mechanisms behind the challenges and consequently evaluate the merits and defects of the recent solutions. We anticipate some compromises on the ideal cloaks are required in order to achieve practical invisibility cloaks in the future.
\end{abstract}

Key words: Transformation optics, Cloak, Metamaterial, Full polarization.

\section{Introduction}

Generally, when the light is incident onto an object, the scattering fields will make the object visible to the observers (Figure 1a). If the total summation of the scattering fields is zero, the object will become invisible. An invisibility cloak is such a device that can totally suppress the scattering of the object. It can smoothly guide the light around the hidden object as if nothing were there (Figure 1b). Usually, an ideal invisibility cloak in people's mind is: zero scattering, omnidirectional, broadband, phase preserved, large scale, independent of the hidden objects and of environment background, and polarization-insensitive.

With the development of metamaterials $[1,2]$, it is scientifically possible to realize the invisibility, which has attracted great research interests in electromagnetic community. In the past few years, scientists have made remarkable efforts to achieve invisibility. In 2005, Alù and Engheta attempted to achieve the invisibility with the method of scattering cancellation [3]. By adopting plasmonic materials coated on an object, the total scattering of these two objects together can be cancelled and hence these two objects as a whole can be invisible. The cloak designed by this method is dependent of the hidden

\footnotetext{
*e-mail: hansomchen@zju.edu.cn
}

object. As this method only consider suppressing the dipole mode, it is limited to sub-wavelength scale. In 2006, Pendry et al. [4] and Leonhardt [5] independently proposed their invisibility cloak design strategies. Utilizing the "metric invariance" of Maxwell's equations, Pendry et al. proposed to create a "hole" in physical space based on a coordinate transformation. In Pendry et al.'s method, light can be smoothly guided around the hidden object and propagate through the cloak without any perturbation. On one hand, the advantages of this method is that both path and phase of light are preserved, and therefore the cloak based on this method is very close to the ideal invisibility cloak in people's mind. On the other hand, due to the phase preservation requirement, light propagates with a superluminal phase velocity in the cloak, and thus the bandwidth is limited for a cloak working in air environment [6]. In Leonhardt's cloak, a complex plane with properly chosen branch cuts and Riemann sheets is adopted $[5,7]$. It is possible to get rid of the superluminal velocity of electromagnetic waves in the cloak by virtue of non-Euclidian transformation, for the reason that the phase will not be preserved. Therefore, Leonhardt's cloak is a ray-optics cloak rather than a wave cloak. As the design and realization of both the Pendry et al.'s cloak [4] and Leonhardt's cloak [5] are associated with very rigorous requirements, the experimental investigations are very rare compared with plenty of theoretical 
(a)

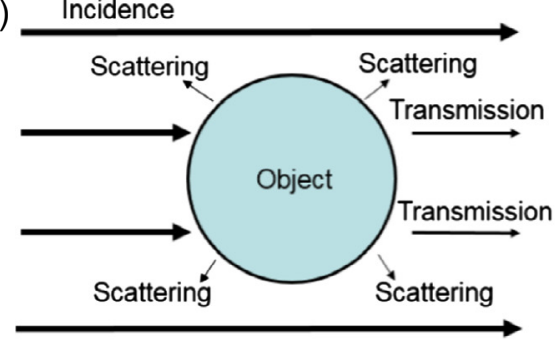

(b)

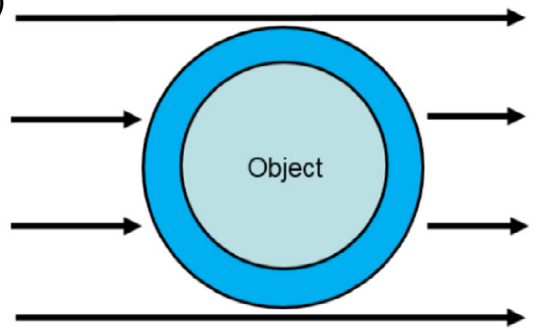

Figure 1. (a) The scheme that an object is illuminated with light from left. When light is incident onto an object, the scattering fields will make the object visible to observers. (b) An invisibility cloak can totally suppress the light scattering and guide light around the hidden object, as if nothing were there.

studies. In particular, achieving an invisibility cloak in reality is a big challenge. It should be noted that the transformation optics not just can be applied to invisibility cloak, actually, it is a powerful tool for designing a wide variety of optical devices, such as lens [8-11], concentrator [12], rotator [13, 14], bending waveguide [15, 16], optical black hole [17], et al., and we refer the reader to some recent reviews [18-20].

In this paper, we start from the theory of transformation optics and review the challenges and the recent progress in the invisibility cloaks from a practical perspective. In particular, we mainly focus on challenges of pushing invisibility cloaks towards reality and experimental investigations. Particular stress is laid on the following main challenges:

- non-extreme parameters,

- homogeneity,

- omnidirectivity,

- full polarization,

- broad band.

We analyze the physical mechanisms behind these challenges and evaluate the merits and defects of the recent solutions. At the end, we provide our viewpoint for designing practical invisibility cloaks. Representative but non-exhaustive references are listed in a table.

\section{Transformation optics: guiding light to make objects invisible}

Assume the lines of electric or magnetic force are fixed to the coordinates. Thus when the coordinate system is distorted, the lines of force are also distorted, which means we can transform the coordinate system to another one to reshape the trajectories of the fields [20]. The coordinate system before transformation is defined as virtual space, and the one after transformation defined as physical space. Due to the metric invariance of the Maxwell's equations [4], in the physical space or transformed space, the form of Maxwell's equations maintain the same, only the values of permittivity and permeability are changed. Let's start from Maxwell's equations [21]. Before the transformation, the equations are:

$$
\nabla \times \bar{H}=\overline{\bar{\varepsilon}} \frac{\partial}{\partial t} \bar{E}, \nabla \times \bar{E}=-\overline{\bar{\mu}} \frac{\partial}{\partial t} \bar{H} .
$$
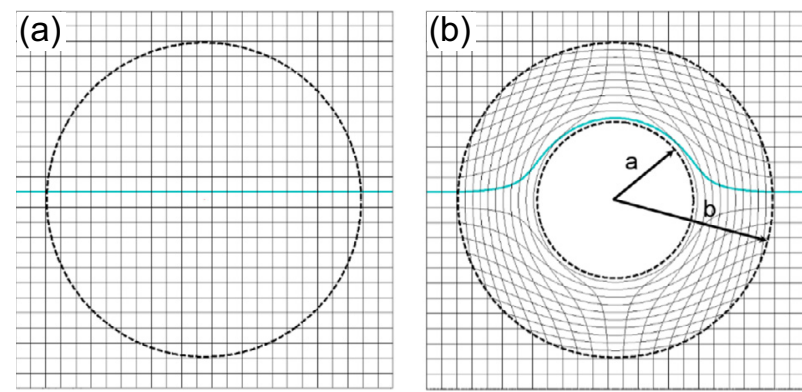

Figure 2. A circle in the virtual coordinate space (a) is transformed to an annulus in the physical coordinate space (b). The center point in the virtual space is therefore transformed to a circle (the small circle) in the physical space. The small circle and the big circle are the internal and external boundaries of the cloak respectively. The green lines represent the trajectories of light.

If we apply a coordinate transformation $\left(x_{1}^{\prime}, x_{2}^{\prime}, x_{3}^{\prime}\right)=f\left(x_{1}, x_{2}, x_{3}\right)$, the equations maintain the same forms in the new coordinate system [4, 22]:

$$
\nabla \times \bar{H}^{\prime}=\overline{\bar{\varepsilon}}^{\prime} \frac{\partial}{\partial t} \bar{E}^{\prime}, \nabla \times \bar{E}^{\prime}=-\overline{\bar{\mu}}^{\prime} \frac{\partial}{\partial t} \bar{H}^{\prime} .
$$

Constitutive parameters in the new coordinate $\overline{\bar{\varepsilon}}^{\prime}, \overline{\bar{\mu}}^{\prime}$ can be expressed by original ones $\overline{\bar{\varepsilon}}, \overline{\bar{\mu}}$ :

$$
\overline{\bar{\varepsilon}}^{\prime}=\frac{J \overline{\bar{\varepsilon}} J^{T}}{\operatorname{det}(J)}, \quad \overline{\bar{\mu}}^{\prime}=\frac{J \overline{\bar{\mu}} J^{T}}{\operatorname{det}(J)},
$$

where $J\left(J_{i j}=\partial x_{i}^{\prime} / \partial x_{j}(i, j=1,2,3)\right)$ is the Jacobean transformation matrix between the physical space and the virtual space.

Let's take a cylindrical cloak for example [23, 24]. The scheme of the cylindrical cloak is to transform a circle with $0<\rho<b$ in the virtual space to an annulus with $a<\rho^{\prime}<b$ in the physical space, as shown in Figure 2. The center point in the virtual space is therefore transformed to a circle (the hidden region) in the physical space. The transformation equations between two spaces are as follows:

$$
\rho^{\prime}=\frac{(b-a)}{b} \rho+a, \quad \theta^{\prime}=\theta, z^{\prime}=z,(0<\rho<b),
$$

where $\rho^{\prime}, \theta^{\prime}, z^{\prime}$ are the radial, angular and vertical coordinates in the coordinate physical space respectively, and $\rho, \theta, z$ are the radial, angular and vertical coordinates in the virtual coordinate 
space respectively. Using the coordinate transformation method, we can get the constitutive parameters of the cloak in the physical space (in the following, we drop the primes for aesthetic reasons):

$$
\begin{aligned}
\varepsilon_{\rho} & =\mu_{\rho}=\frac{(\rho-a)}{\rho}, \quad \varepsilon_{\theta}=\mu_{\theta}=\frac{\rho}{(\rho-a)}, \quad \varepsilon_{z}=\mu_{z} \\
& =\left(\frac{b}{(b-a)}\right)^{2} \frac{(\rho-a)}{\rho}, \quad(a<\rho<b) .
\end{aligned}
$$

In the original coordinate system, the light rays go straight from left to right (Figure 2a). While in the transformed coordinate system, the light rays fixed to the coordinate now are bent around the cloak, thus the objects inside cloak are undetectable (Figure 2b).

\section{Challenges and progress}

The transformation optics provides a very powerful tool to design the invisibility cloaks, but the constitutive parameters of these theoretically ideal cloaks are difficult to achieve. Take a glance at the constitutive parameters of an ideal cylindrical cloak (Eq. (5)), one can find that this cloak requires both electric and magnetic anisotropic materials and the relative permittivity and permeability should be ideally equal to each other. This is the first challenge for fabricating a cloak. The reason is that, to achieve identical electric and magnetic constitutive parameters, it is necessity to use the extremely fine nanofabrication for metamaterials and is very difficult to implement with the current technologies. The second challenge is that the values of the parameters are space dependent in radial direction, i.e., the materials of the cloak are inhomogeneous. In the experimental realization, this continuously varying parameters need to be discretized, but the discretization will deteriorate the invisibility performance [25]. If look into further, one can find the third challenge is that, the constitutive parameters contain extreme values, for example, at $\rho=a, \varepsilon_{\theta}$ and $\mu_{\theta}$ reach infinite, and in the cloaking region $(a<\rho<b)$, these parameters should cover all the values in the range from 0 to $\infty$. This stringent requirement becomes looser for a spherical cloak [4], where the constitutive parameters only need to cover all the values in the range from 0 to 1 . However, implementation of all of these parameters is still a big challenge. To overcome these challenges, both theoretical and experimental works have been investigated in the past few years. In the following, we will review the physical mechanisms behind these challenges, theoretical solutions and experimental investigations, from a practical perspective.

\subsection{From ideal cloak to practical cloak: removing extreme parameters requirement}

As extreme parameters hinder the experimental realization of the invisibility cloaks, removing such parameters but still keeping acceptable invisibility performance is the first task scientists need to face when pushing the cloak from an ideal one to a practical one. The physical mechanism behind this challenge is that the coordinate transformation is from a line/point in the virtual space to a surface in the physical space at the inner boundary of the cylindrical/spherical cloak.

Let's first take the cylindrical cloak for example, as the $z$ component electromagnetic fields are nonzero everywhere except in the hidden region, electric and magnetic surface currents along the $\theta$ direction should exist at the inner boundary of the cloak in order to satisfy the discontinuity of the tangential $z$ component of electromagnetic fields. This requires both $\varepsilon_{\theta}$ and $\mu_{\theta}$ to be infinite in order to generate the surface currents [26, 27]. Besides, when electromagnetic waves propagate along $z$ direction, the phase velocity of the waves propagating in the cloak should be equal to those propagating in air, which will require $n_{z}=\sqrt{\varepsilon_{\theta} \mu_{\rho}}=\sqrt{\mu_{\theta} \varepsilon_{\rho}}=1$. As $\varepsilon_{\theta}=\mu_{\theta}=\infty$, one can see $\varepsilon_{\rho}, \mu_{\rho}$ have to be zero. Note that as this transformation is phase preserved, i.e. the phase at the inner boundary of the cloak should be the same as the counterpart at the point in virtual space, the phase velocity at the inner surface should be infinite, i.e. $n_{\theta}=\sqrt{\varepsilon_{\rho} \mu_{z}}=\sqrt{\mu_{\rho} \varepsilon_{z}}=0$. This requires either (or both) of the $\rho$ and $z$ components of the permittivity and permeability to be zero in order to satisfy this phase preservation. It should also be noted that $\varepsilon_{\rho}=\mu_{\rho}=0$ is a necessary condition in order to meet the phase preservation requirement, while the values of $\varepsilon_{z}, \mu_{z}$ don't have to equal 0 . For example, with certain transformation functions, $\varepsilon_{z}, \mu_{z}$ can be kept as nonzero constants everywhere in the cloak [28].

For the spherical cloak, the transformation is from a point to a spherical surface. The fields cannot be decomposed into the transverse electric and transverse magnetic fields. Because the tangential fields (the $\theta$ and $\varphi$ components of the electromagnetic fields) at the inner surface of the spherical cloak are counterparts of the center point in the virtual space, which is a singularity so that the $\theta$ and $\varphi$ components of the electromagnetic fields cannot be defined in the spherical coordinate system, they have to be zero. Thus the boundary condition of the tangential fields at the inner surface of the spherical cloak is automatically satisfied, and does not require infinite values of the permittivity and permeability. The extreme parameters are not necessary. It doesn't like the cylindrical cloak where the tangential $z$ components of the electromagnetic fields (which can be accurately defined in the center point in virtual space) are discontinuous at the inner surface of the cloak. So in the spherical cloak, only the phase preservation requirements need to be considered. As the phase velocity at the inner surface should be infinite, i.e. $n_{\theta}=\sqrt{\varepsilon_{\varphi} \mu_{r}}=\sqrt{\mu_{\varphi} \varepsilon_{r}}=0$ and $n_{\varphi}=\sqrt{\varepsilon_{\theta} \mu_{r}}=\sqrt{\mu_{\theta} \varepsilon_{r}}=0$, it requires $\mu_{r}=0$ and $\varepsilon_{r}=0$.

Therefore, when consider removing the extreme parameters, we are mostly talking about the cylindrical cloak. To overcome this challenge, one possible approach is to simplify the singular parameters to nonsingular ones by using the eikonal approximation, i.e. altering the impedance but keeping the refractive index unchanged; the other approach is to adopt the so-called carpet cloak strategy.

In the first approach, considering the TE-polarization $\left(E_{z}\right.$ polarization), only three components $\varepsilon_{z}, \mu_{\rho}, \mu_{\theta}$ are involved. Although $\mu_{\theta}$ is singular at $\rho=a$, the index of refraction $\left(\sqrt{\mu_{\theta} \varepsilon_{z}}\right)$ is a constant. Therefore, by retaining the index of refraction unchanged, i.e. the trajectories of reflected and refracted light will stay the same, while altering the impedance, 

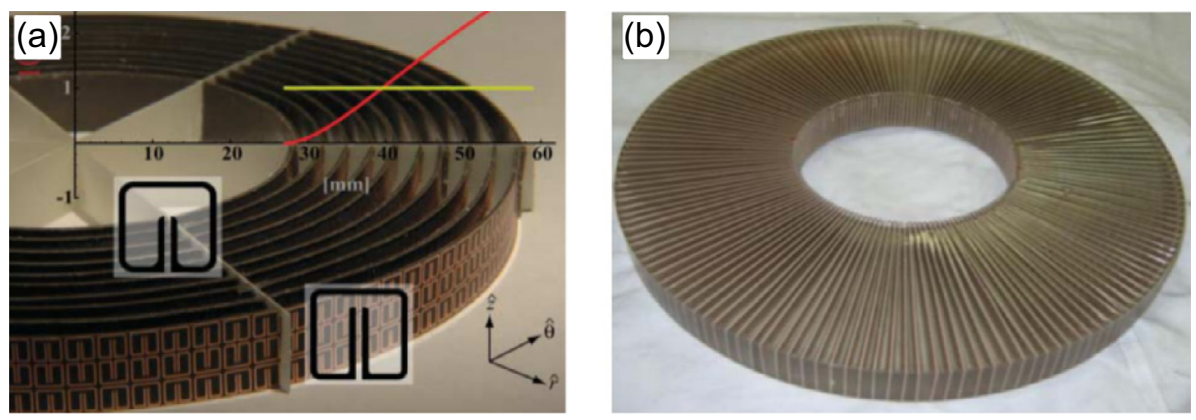

Figure 3. A reduced microwave omnidirectional cloak for (a) TE-polarization wave [24] and (b) TM-polarization wave [31].

(a)
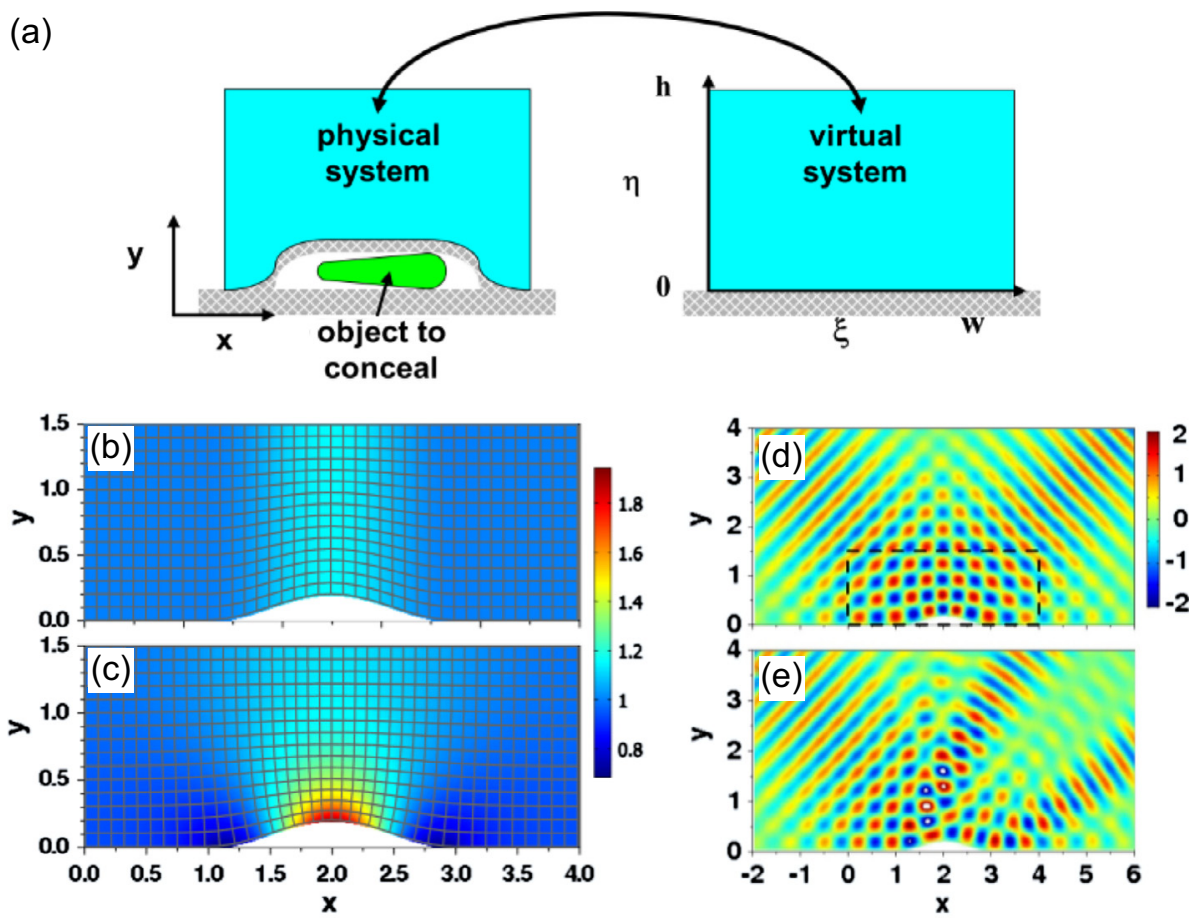

Figure 4. (a) The scheme of quasiconformal mapping carpet cloak. A hidden area in the physical system is mapped into a ground plane in the virtual system. (b) The profile $n^{2}$ after transformation. (c) The replaced profile $n^{2}$ without anisotropy from quasiconformal mapping. (d) Electric field distribution in $z$ direction with cloak. (e) Electric field distribution in $z$ direction without cloak [38].

we can remove the extreme parameters of $\mu_{\theta}$. The parameters of the reduced cloak can be simplified as [23, 24], $\varepsilon_{z}=(b /(b-a))^{2}, \mu_{\rho}=((\rho-a) / \rho)^{2}, \mu_{\theta}=1$. To overcome the inhomogeneity, the cloak is discretized into several homogeneous regions, which are experimentally realized with splitring resonators (SRRs), as shown in Figure 3a. This is also the first experimental demonstration of the transformation optics based cloak. A similar concept was extended to TM-polarization $\left(H_{z}\right.$ polarization) [29-33], where the constitutive parameters can be reduced as $[29,31,34], \mu_{z}=1, \varepsilon_{\theta}=((b-a) / a)^{2}$, $\left.\varepsilon_{\rho}=((b-a) / a)^{2}(\rho-a) / \rho\right)^{2}$. Besides, this cloak is nonmagnetic, thus it can be applied to optical spectrum [34]. The performance of the reduced-parameter cloak was experimentally demonstrated in microwave frequency (Figure 3b). The experimental measurement results of both reduced cloaks show that the forward and backward scattering strongly decrease. However, due to simplification, these cloaks will still lead to considerable scattering, which can be improved by some higher order transformation functions [30, 32, 33].

Another way to eliminate the extreme parameters is to adopt the coordinate transformation from a surface in the virtual space to another surface in the physical space, which is the so-called carpet cloak [35-37]. The carpet cloak concept was first proposed by Li and Pendry in 2008, as shown in Figure 4 [38], which can render an object invisible on a reflective surface. Physically, the two surfaces in different spaces only change the scale in a finite domain, so the constitutive parameters are non-extreme. In order to simplify the parameters further, quasiconformal mapping is applied to minimize the anisotropy of the materials. By replacing these materials with isotropic ones, a cloak consisting of non-extreme and isotropic materials is achieved. When embedding the quasiconformal mapping carpet cloak in a certain background, all parameters of the cloak are isotropic and greater than one, leading to broad 

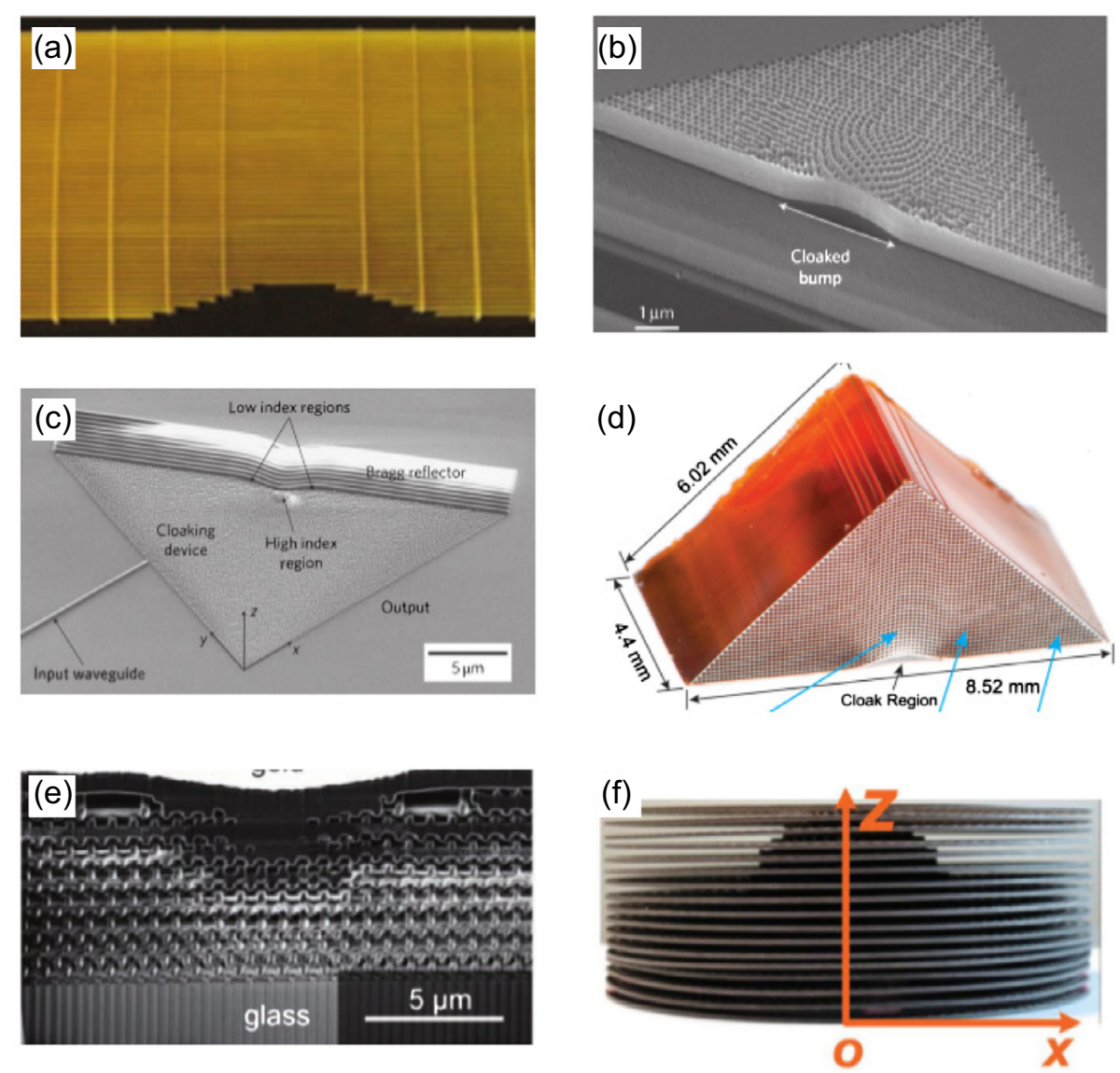

Figure 5. Quasiconformal mapping based carpet cloaks. (a) A 2D microwave carpet cloak [42]. (b)-(c) 2D optical carpet cloaks [43, 44]. (d) A 2D terahertz carpet cloak [45]. (e) A 3D optical carpet cloak [52]. (f) A 3D microwave carpet cloak [53].

bandwidth and low loss. We should note that the carpet cloak concept has also been extended for surface wave [39-41].

The first experimental verification of quasiconformal mapping carpet cloak has been carried out by Liu et al. [42]. This carpet cloak is composed of nonresonant metamaterial elements working in microwave frequency (Figure 5a). Later, several similar cloaks with different operating frequencies have been also realized. With the aid of nanotechnology, reference [43] (Figure 5b) and reference [44] (Figure 5c) experimentally demonstrate optical quasiconformal mapping carpet cloaks independently. In reference [43], holes in silicon with varying density are designed to define the effective refraction index required. While in reference [44], nanometer-size silicon structures etching in the SOI wafer yield spatial effective refraction index profile. A similar carpet cloak working at terahertz frequency has been also realized in reference [45], which is composed of polymer with different-size holes (Figure 5d). More quasiconformal mapping based carpet cloaks have been realized in microwaves [46-49] and visible light spectrum [50,51].

Unlike the 2D cloaks, most of which usually work for certain polarization in a plane, the 3D cloaks inherently work for full polarization in a 3D space. Two 3D isotropic carpet cloaks with different operational frequencies have been realized recently. In these cloaks, polarization-insensitive metamaterials to yield spatially varying refraction index are properly designed. The metamaterial elements of the $3 \mathrm{D}$ optical carpet cloak are woodpile photonic crystals with tailored polymer filling fraction in reference [52] (Figure 5e), while their microwave counterpart are drilling inhomogeneous holes in multilayered dielectric plates in reference [53] (Figure 5f). However, the later theoretical research manifests that anisotropy is intrinsically required for a $3 \mathrm{D}$ carpet cloak with rotational symmetry [54]. For the 3D isotropic carpet cloak, when the rays incident with $k_{\phi} \neq 0$, the cloak will not properly redirect the rays [54]. It should be noted that the isotropic 3D carpet cloaks still clearly reduce the scattering of the hidden objects.

Though successfully demonstrated, the quasi-conformal mapping carpet cloaks still have some limitations. Firstly, the spatially varying index of refraction makes the fabrication challenging. Secondly, compared with the cloak shell, the hidden region is too small. Finally, the approximation of replacing the anisotropy materials with isotropic ones will introduce a lateral shift of the scattered waves. This lateral shift is comparable to the height of hidden region, which will render the cloak detectable [55].

Besides these two strategies, another successfully demonstrated method is based on inductor-capacitor transmission line network. In this method, the metamaterials are replaced by lumped elements, which can easily control each component of the constitutive parameters and thus make the full-parameter omnidirectional cloak a reality. However, due to the lumped 
(a)
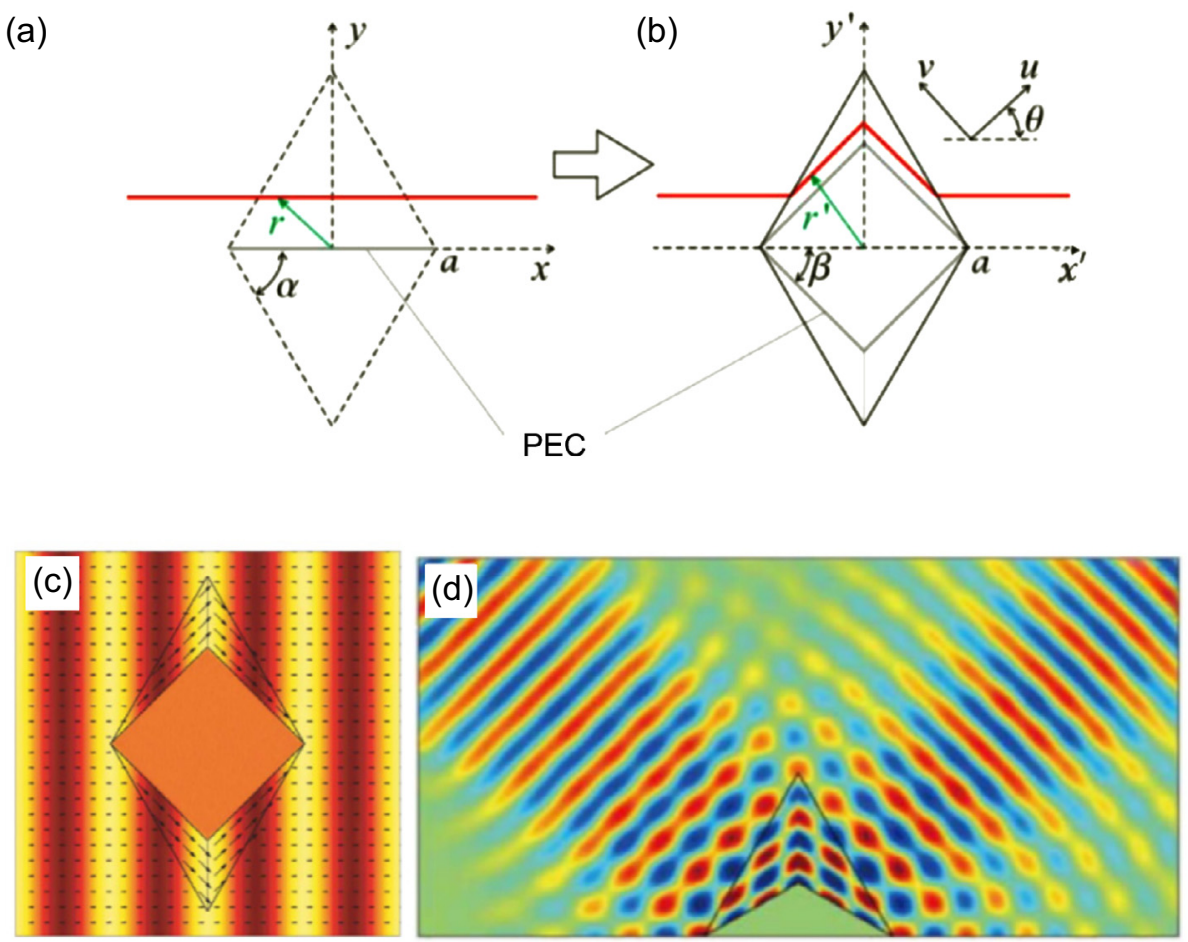

Figure 6. (a)-(b) The scheme of one-dimensional cloak consisting of homogeneous materials [58]. The diamond space in virtual space demonstrated by the angle $\alpha$ is compressed into the space $(\alpha-\beta)$ in physical space. (c) The magnetic field distribution and the poynting vectors when a polarized plane wave is incident onto the perfect one-dimensional cloak [58]. (d) The magnetic field distribution when a polarized plane wave is incident onto the perfect carpet cloak [59].

elements, these cloaks can only work at low frequency, which may limit their practical application $[56,57]$.

\subsection{From wavelength scale cloak to large scale practical cloak: removing inhomogeneity requirement}

Large scale cloaks are requisite for practical applications, for example, hiding a plane, a car, or even a living creature, such as a human being. As we look back to the cloaks mentioned above, one can find that these cloaks are usually with size less than ten-wavelength, owing to the challenge of fabricating large scale inhomogeneous materials. The inhomogeneous parameters result from the nonlinear coordinate transformation. Properly choosing the coordinate transformation may remove the inhomogeneity of the parameters, and therefore makes the large scale invisibility cloaks much easier to realize. The first theoretical cloak with homogeneous parameters was proposed by Sheng et al. [58]. They proposed a linear coordinate transformation strategy to design a one-dimensional cloak, as shown in Figures 6a-6d, wherein the diamond space in the virtual space demonstrated by the angle $\alpha$ is compressed into the space $(\alpha-\beta)$ in the physical space. One can see that a one-dimensional cloak with only one anisotropic material is achieved. Using the same linear transformation, carpet cloak with homogeneous parameters can be also designed with perfect performance (Figure 6d) [59].
By applying the eikonal approximation, the one-dimensional or carpet cloak is further simplified to be nonmagnetic, which can be experimentally implemented by two approaches.

1. One approach is to adopt natural birefringent crystals. Two optical carpet cloaks have been realized with calcite crystals (Figures 7a-7b) [60, 61], which significantly extend the hidden region from several wavelength scale to around 3500 wavelength scale. Later, with a similar concept, a terahertz carpet cloak was realized with sapphire crystals [62].

2. The other approach is to use the artificial materials composed of multilayers of two isotropic materials with different permittivities, which is according to the effective medium theory. Based on this method, an optical carpet cloak has been implemented with uniform silicon grating structures (Figure 7c) [63-65]; while its microwave counterpart has been implemented with multilayer of FR4 dielectric slabs with air spacing (Figure 7d) [66].

These simplified linear transformation based carpet cloaks are usually broadband and low-loss. For example, the optical carpet cloak in reference [60] works at least from 189.9 to 202.7 THz; the operational bandwidth of optical carpet cloak in reference [61] encompasses red, green, and blue light; the terahertz carpet cloak in reference [45] operates very well from 0.2 to $1.0 \mathrm{THz}$; and the microwave carpet cloak in reference 

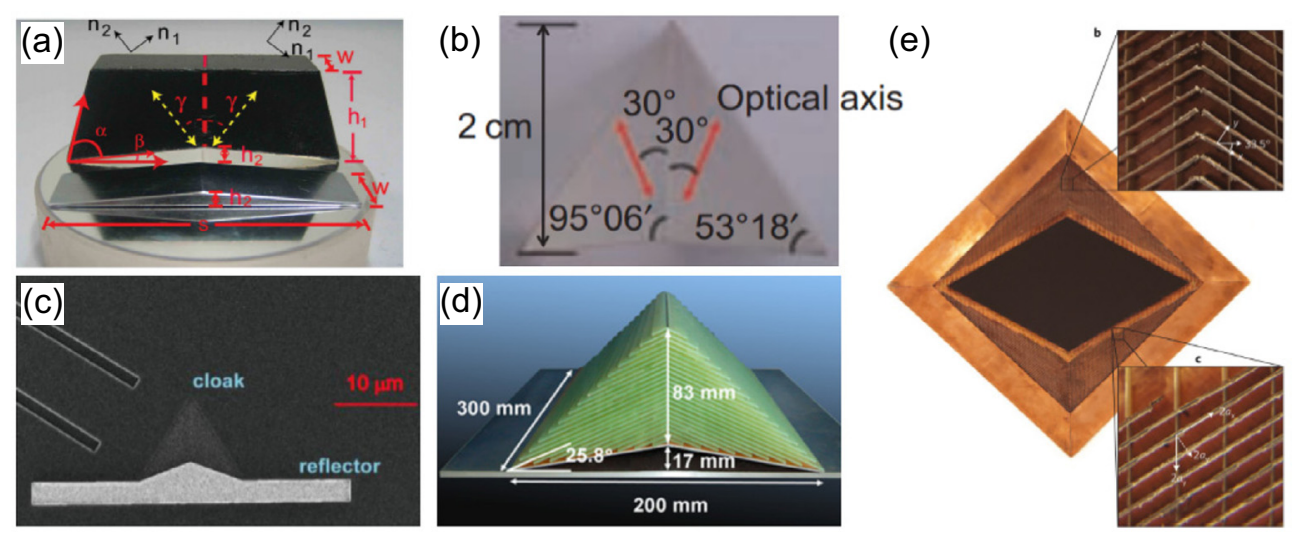

Figure 7. Linear coordinate transformation based cloaks. (a)-(b) Large scale optical carpet cloaks consisting of natural birefringent materials [60, 61]. (c) An optical carpet cloak composed of multilayers [65]. (d) A microwave carpet cloak composed of mutlilayers [66]. (e) A one-dimensional full-parameter microwave cloak [67].

[42] works at frequencies from 8 to $18 \mathrm{GHz}[45,60,61]$. Moreover, the cloaks realized by these two approaches have their own advantages. For linear transformation based carpet cloaks, the ratio of the size of the hidden region to that of the cloak relates to the anisotropy of the material. The larger ratio corresponds to the larger anisotropy. Thus the merit of the second approach lies in the larger anisotropy of artificial materials compared with natural materials [65]; while the benefit of the first approach is that it is convenient to fabricate a macroscopic cloak with natural materials [61]. As simplified parameters are utilized, these cloaks sacrify the impedance matching at the boundary between background and cloak, resulting in some reflections.

Because the whole carpet cloak is equivalent to a PEC/PMC sheet, the observer above the sheet can't differentiate the cloak from the PEC/PMC sheet. However, if the carpet cloak isn't sitting on a PEC/PMC sheet, for example, hanging in the air, only from the direction paralleling the PEC/PMC sheet will the observer not see the cloak. He will see a levitating PEC/PMC slice from other directions. This indicates the carpet cloak is equivalent to a one-directional cloak for hiding isolate objects.

Recently, a full-parameter of such one-dimensional cloak is realized with homogeneous metamaterials (Figure 7e) [67]. Unlike the simplified cloaks, it is approximation-free, which is a substantial improvement on the scattering suppression. In this cloak, double-side SRRs are utilized to couple to $\mu_{y}$ $\left(\mu_{y}<1\right)$ and $\varepsilon_{z}\left(\varepsilon_{z}>1\right)$, and corrugations to provide $\mu_{x}$ $\left(\mu_{x}>1\right)$. This cloak shows the good performance promised by transformation optics at frequency of $10.2 \mathrm{GHz}$. Due to the magnetic resonant elements, the cloak is still limited to a narrow band.

\subsection{From unidirectional cloak to multi-directional and omnidirectional, broadband, and polarization-insensitive practical cloak: removing the phase preservation requirement}

To achieve a practical cloak, omnidirectivity (multidirectivity), broad band, and polarization-insensitivity are very important. Strategies based on linear coordinate transformation can be further developed to get omnidirectional cloaking performance [68-70]. Therefore, an omnidirectional cloak composed of homogeneous materials, providing convenience for large scale industrial fabrication, becomes a possibility. Chen and Zheng proposed such a polygonal cloak, wherein the cloak is divided into several segments, which can be grouped into two types (region I and region II), and in each segment a linear coordinate transformation is applied along axis of its own coordinates (Figures 8a-8b) [68]. As the concealed region (the white area in Figure 8b) in the physical space is transformed from an ultra small one (the white area in Figure 8a) in the virtual space, objects hidden in this region are hard to see by naked eyes. However, because the parameters of region I in the physical space are very extreme, it is still very difficult to realize this cloak. One solution is to simplify the cloak by removing the region I medium and only using region II medium. This simplified cloak is therefore composed of only one anisotropic material (Figure 8c). The omnidirectional performance of the ideal cloak is sacrificed with this simplification. By utilizing calcite, a natural anisotropic material, a multidirectional TM-polarization visible light cloak working for six incident directions is obtained (Figure 8e). As anisotropic material is required in this cloak, it works for only one polarization.

To achieve a polarization-insensitive broadband optical cloak, it requires isotropic materials before both electrical and magnetic anisotropic metamaterials can be achieved. As the phase lines in the isotropic materials will be different from those in the anisotropic materials of an ideal cloak, one has to abolish the phase preservation requirement. Indeed, the phase preservation requirement is not necessary in visible light spectrum for the reason that, human eyes are insensitive to the phase and polarization of light [71]. Therefore Chen et al. proposed a broadband stand-free ray-optics cloak, as shown in Figure 8d. Unlike the polarization-sensitive polygonal cloak, wherein the phase lines are not perpendicular to the ray propagation direction, in this natural light cloak the two types of anisotropic parameters are replaced by isotropic 

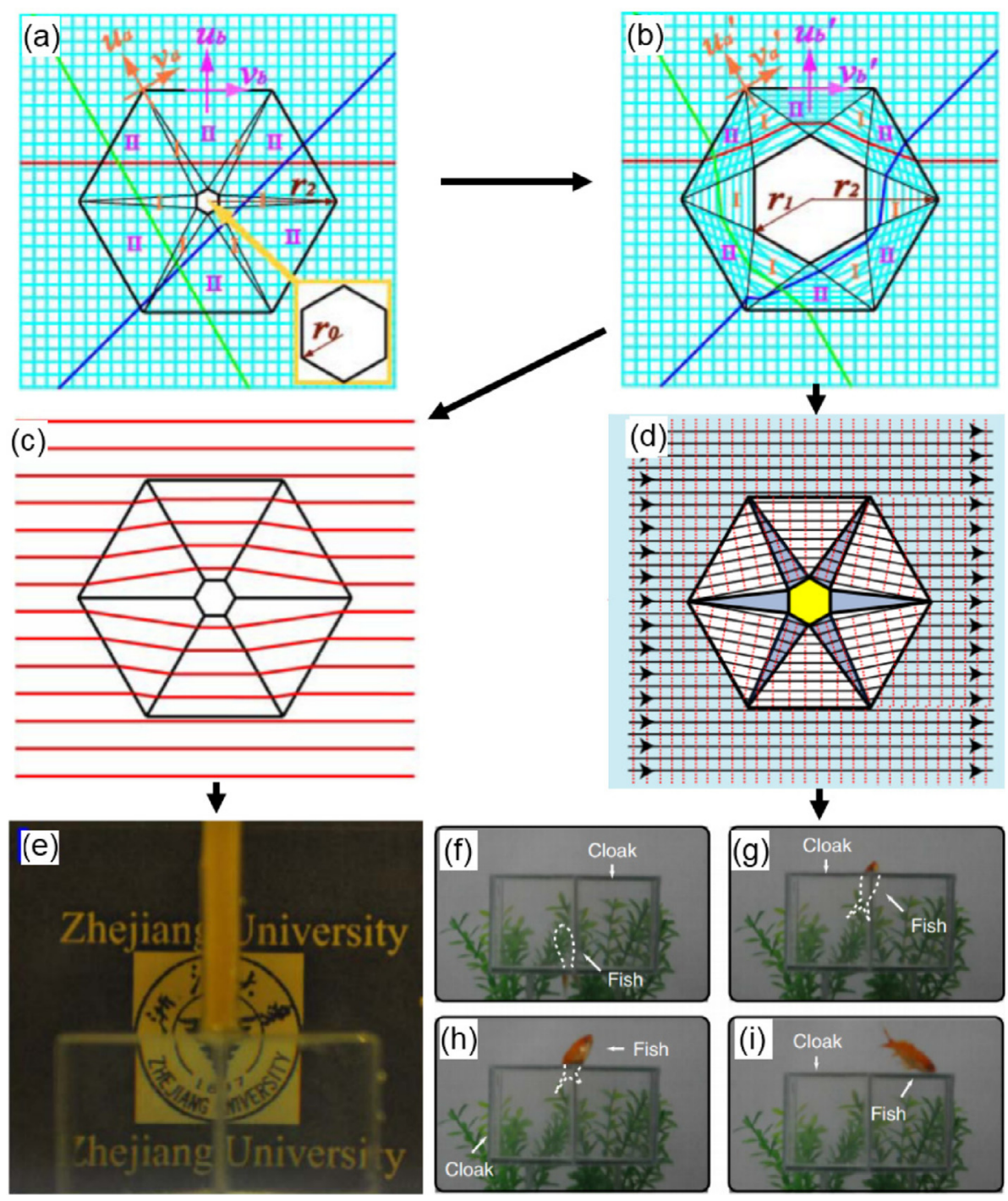

Figure 8. (a)-(b) The scheme of an omnidirectional hexagonal cloak consisting of homogeneous materials. The cloak is divided into several segments, grouped into two types (region I and region II), and in each segment a linear coordinate transformation is applied along axis of its own coordinates. (c) The scheme of a multi-directional polarization-sensitive hexagonal cloak [68]. (d) The scheme of a multi-directional polarization-insensitive hexagonal cloak [71]. (e) A multi-directional, broadband, polarization-sensitive, and large scale practical cloak [68]. (f)-(i) A multi-directional, broadband, polarization-insensitive, and large scale practical cloak [71].

ones and therefore the cloaking performance is full polarization. With the aid of homogeneous, isotropic and commonly available materials, such as water, glass and air, a multi-directional polygonal cloak is obtained (Figures $8 \mathrm{f}-8 \mathrm{i}$ ). In this cloak, living creatures, such as fish and cat can be hidden in plain sight for several incident directions with broad operational bandwidth. The experiment results show that when the oblique view angle is larger than $\pm 3^{\circ}$, the image distortion is observable. Pushing this cloak further to an omnidirectional one is still a big challenge.

\section{Discussion}

Besides the transformation optics method, some other cloaking strategies, such as scattering cancellation, ray-optics conformal mapping, and some hybrid methods, have been also developed. These strategies show their specific merits and limitations when designing large scale practical cloaks.

The scattering cancellation method is to reduce the total scattering cross section of spherical or cylindrical dielectric objects by covering them with plasmonic materials. The physical mechanism of this method is that the dipole moment of hidden dielectric object and that of plasmonic cloak shell cancel each other [3]. The parameters of scattering cancellation based cloaks are homogeneous and helpful for experimental implementation. Some experimental verifications were carried out at microwave frequencies, for example, the plasmonic cloak [72, 73], the three-dimensional plasmonic cloak [74], and the so-called "mantle cloak" [73, 75]. The advantage of the method is that the total scattering of the cloak, which represents the cloaking performance, can be quantitatively calculated $[3,76]$. The limitation is that, when the size of the hidden 
Table 1. Properties of experimentally realized electromagnetic cloaks

\begin{tabular}{|c|c|c|c|c|c|c|c|c|}
\hline Reference & $\begin{array}{c}\text { Broad } \\
\text { band }\end{array}$ & $\begin{array}{c}\text { Air } \\
\text { background }\end{array}$ & $\begin{array}{c}\text { Phase } \\
\text { preservation }\end{array}$ & Omnidirectivity & $\begin{array}{c}\text { Full } \\
\text { polarization }\end{array}$ & $\begin{array}{c}\text { Object } \\
\text { independence }\end{array}$ & $\begin{array}{c}\text { Operational } \\
\text { frequency }\end{array}$ & $\begin{array}{l}\text { Size of } \\
\text { objects }\end{array}$ \\
\hline Ref. [72] (2009) & No & Yes & Yes & Yes & No & No & Microwave & $\sim 0.25 \lambda$ \\
\hline Ref. [24] (2006) & No & Yes & Yes & Yes & No & Yes & Microwave & $\sim 1.5 \lambda$ \\
\hline Ref. [42] (2009) & Yes & No & No & No & No & Yes & Microwave & $\sim 0.5 \lambda$ \\
\hline Ref. [43] (2009) & Yes & No & No & No & No & Yes & Optical & $\sim 0.3 \lambda$ \\
\hline \multicolumn{9}{|l|}{ Ref. [44] (2009) } \\
\hline Ref. [45] (2011) & Yes & No & No & No & No & Yes & Terahertz & $\sim 0.4 \lambda$ \\
\hline Ref. [49] (2013) & Yes & Yes & No & No & No & Yes & Microwave & $\sim 0.24 \lambda$ \\
\hline Ref. [65] (2011) & Yes & No & Yes & No & No & Yes & Optical & $\sim 1.2 \lambda$ \\
\hline Ref. [66] (2011) & Yes & No & Yes & No & No & Yes & Microwave & $\sim 1 \lambda$ \\
\hline Ref. [78] (2012) & Yes & Yes & No & Yes & No & Yes & Microwave & $1 \lambda$ \\
\hline Ref. [77] (2013) & Yes & No & Yes & No & No & Yes & Microwave & $\sim 1.2 \lambda$ \\
\hline \multicolumn{9}{|l|}{ Ref. [60] (2011) } \\
\hline Ref. [68] (2012) & Yes & No & No & No & No & Yes & Optical & $\sim 5 \times 10^{3} \lambda$ \\
\hline Ref. [71] (2013) & Yes & Yes & No & No & Yes & Yes & Optical & $\sim 5 \times 10^{5} \lambda$ \\
\hline
\end{tabular}

object increases, higher dipole modes need to be suppressed, making it too complicated to implement. Therefore, this method is usually applicable in sub-wavelength scale. Moreover, being different from the transformation optics method that is applicable to any arbitrary shaped cloaks, the scattering cancellation method is so far limited in some regular shaped cloaks, e.g. cylindrical, spherical, and ellipsoidal cloaks, etc. This is because only in these regular shaped cloak structures can one find analytical scattering solutions.

The conformal mapping method $[5,77]$ is used to design invisibility cloaks, by mapping a complex plane in the original space to a stack of Rieman sheets and branch cuts in the transformed space. In the conformal mapping, a small square in the virtual space is transformed to another square in the physical space, thus the constitutive parameters are isotropic. As the parameters of the cloaks are inhomogeneous and vary in a large range, realization of these cloaks is still a big challenge. These cloaks can be made to be broadband due to the fact that phase of the light is inherently not preserved. The stringent requirement for conformal mapping based cloaks is looser than that for the transformation optics based cloaks, where the phase should be rigorously preserved. From this point, one can see the transformation optics cloaks can cloak waves, while the conformal mapping cloaks can only cloak rays.

An interesting way to design invisibility cloaks is to combine the merits of the transformation optics, conformal mapping and scattering cancellation together. In the hybrid method in reference [78], an optimization scheme based on scattering cancellation is used to design the parameters of the cloak; anisotropic constitutive parameters are used, which is a fundamental property of the transformation optics; and nonsuperluminal propagation of electromagnetic waves is inherited, which is a superior advantage of non-Euclidian transformation cloaks constructed with complex branch cuts. Using only a few layers, a one wavelength cloak with a relatively broad bandwidth has been achieved.

It should be noted that the stringent requirements for an ideal cloak may be bypassed when considering some specific applications. For example, the superluminal phase propagation does not need be to considered for the diffusive-light cloak [79]; the dc magnetic cloaks [80-82] and dc current cloaks [83-86] work for static fields, so phase preservation requirement and bandwidth issue are not applicable. The electromagnetic surface waves [87] cloak works for surface waves and so it does not need to consider the bandwidth and omnidirectional requirements. The temporary cloak works for a specific time [88], etc. These extended cloaks may find feasible applications for specific purpose.

In Table 1, we summarize some representative but nonexhaustive experimental works on electromagnetic cloaks. From the table, one can see that all successful broadband cloak designs $[42-45,49,52,53,60,61,65,66,68,71,77,78]$ are either abolishing the phase preservation requirement $[68,71$, 78 ] or working in a background medium [42-44, 49, 52, 53, $60,61,65,66,77]$ Successful omnidirectional cloaks achieved so far are still limited in several wavelength scale [24, 72, 73, 78]. Cloak designs for full polarization $[50,52,53,71]$ are very rare. All these cloaks realized so far are designed with isotropic materials, through which full polarization is achieved by sacrifying the phase preservation performance. Another way to achieve full polarization cloak with phase preservation is to design both electric and magnetic anisotropic metamaterials. As it requires fine nanofabrication techniques, a full polarziation cloak realized with both electrical and magnetical anistropic metamaterials is still a challenging work with current technologies and hasn't been demonstrated yet.

As analogs of electromagnetic cloaks, due to their potential application, the acoustic cloaks also receive increasing interest 
[89-92]. Compared with the electromagnetic cloaks, one advantage of the acoustic cloaks is that the bandwidth of electromagnetic cloaks will be limited by the causality, while the acoustic cloaks will not suffer from such limitation. Because the speed of the acoustic wave or linear surface wave is much slower than that of light. With the aid of acoustic metamaterials, some acoustic cloaks have been realized, including scattering cancellation based acoustic cloak [93], 2D omnidirectional cloak [94, 95], 2D carpet cloak [96], and 3D carpet cloak [97], etc. We should note that the concept of cloak has also been extended to the other physical systems, such as thermal fields [98-101], mechanical fields [102] and waves [103], matter waves [104], etc.

\section{Conclusions}

In conclusion, this paper provides a comprehensive overview on recent progress in invisibility cloaks from a practical perspective, where we aim at zero scattering, omnidirectional, large scale, full polarization and broadband practical cloaks, which also conform to that in people's mind. Particularly, the paper emphasises the key issues including non-extreme parameters, homogeneity, omnidirectivity, polarization insensitivity, and broad band. The physical mechanisms behind the stringent requirement of the ideal cloak are discussed. We anticipate that relaxing the stringent requirements for an ideal cloak is a feasible direction to convert the cloaks into practical applications. Moreover, finding some particular applications where the stringent requirements for an ideal cloak may be bypassed is also a very promising work in the future.

Acknowledgements. This work was sponsored by the National Natural Science Foundation of China under Grants No. 61322501, and No. 61275183, the National Program for Special Support of TopNotch Young Professionals, the Program for New Century Excellent Talents (NCET-12-0489) in University, the K.P. Chao's High Technology Development Foundation, and the Fundamental Research Funds for the Central Universities (2014XZZX003-24). We thank H.A. Madni for a critical reading of the manuscript.

\section{References}

1. H. Chen, Metamaterials: constitutive parameters, performance, and chemical methods for realization, Journal of Materials Chemistry 21 (2011) 6452-6463.

2. Y. Liu, X. Zhang, Metamaterials: a new frontier of science and technology, Chemical Society Reviews 40 (2011) 2494-2507.

3. A. Alù, N. Engheta, Achieving transparency with plasmonic and metamaterial coatings, Physical Review E 72 (2005) 016623.

4. J.B. Pendry, D. Schurig, D.R. Smith, Controlling electromagnetic fields, Science 312 (2006) 1780-1782.

5. U. Leonhardt, Optical conformal mapping, Science 312 (2006) 1777-1780.

6. U. Leonhardt, T. Tyc, Broadband invisibility by non-Euclidean cloaking, Science 323 (2009) 110-112.

7. U. Leonhardt, Notes on conformal invisibility devices, New Journal of Physics 8 (2006) 118-118.
8. W.X. Jiang, T.J. Cui, H.F. Ma, X.M. Yang, Q. Cheng, Layered high-gain lens antennas via discrete optical transformation, Applied Physics Letters 93 (2008) 221906.

9. N. Kundtz, D.R. Smith, Extreme-angle broadband metamaterial lens, Nature materials 9 (2010) 129-132.

10. M. Tsang, D. Psaltis, Magnifying perfect lens and superlens design by coordinate transformation, Physical Review B 77 (2008) 035122.

11. M. Yan, W. Yan, M. Qiu, Cylindrical superlens by a coordinate transformation, Physical Review B 78 (2008) 125133.

12. M. Rahm, et al., Design of electromagnetic cloaks and concentrators using form-invariant coordinate transformations of Maxwell's equations, Photonics and Nanostructures Fundamentals and Applications 6 (2008) 87-95.

13. H. Chen, C.T. Chan, Transformation media that rotate electromagnetic fields, Applied Physics Letters 90 (2007) 241105.

14. H. Chen, et al., Design and experimental realization of a broadband transformation media field rotator at microwave frequencies, Physical Review Letters 102 (2009) 183903.

15. J. Huangfu, et al., Application of coordinate transformation in bent waveguides, Journal of Applied Physics 104 (2008) 014502 .

16. M. Rahm, S.A. Cummer, D. Schurig, J.B. Pendry, D.R. Smith, Optical design of reflectionless complex media by finite embedded coordinate transformations, Physical Review Letters 100 (2008) 063903.

17. E.E. Narimanov, A.V. Kildishev, Optical black hole: Broadband omnidirectional light absorber, Applied Physics Letters 95 (2009) 041106.

18. H. Chen, C.T. Chan, P. Sheng, Transformation optics and metamaterials, Nature Materials 9 (2010) 387-396.

19. Y. Liu, X. Zhang, Recent advances in transformation optics, Nanoscale 4 (2012) 5277-5292.

20. J.B. Pendry, A. Aubry, D.R. Smith, S.A. Maier, Transformation optics and subwavelength control of light, Science 337 (2012) 549-552.

21. G.W. Milton, M. Briane, J.R. Willis, On cloaking for elasticity and physical equations with a transformation invariant form, New Journal of Physics 8 (2006) 248-248.

22. U. Leonhardt, Transformation optics and the geometry of light, Progress in Optics 53 (2008) 69-152.

23. S.A. Cummer, B.-I. Popa, D. Schurig, D.R. Smith, Full-wave simulations of electromagnetic cloaking structures, Physical Review E 74 (2006) 036621.

24. D. Schurig, et al., Metamaterial electromagnetic cloak at microwave frequencies, Science 314 (2006) 977-980.

25. Y. Huang, Y. Feng, T. Jiang, Electromagnetic cloaking by layered structure of homogeneous isotropic materials, Optics express 15 (2007) 11133-11141.

26. B. Zhang, H. Chen, B.-I. Wu, J. Kong, Extraordinary surface voltage effect in the invisibility cloak with an active device inside, Physical Review Letters 100 (2008) 063904.

27. B. Zhang, et al., Response of a cylindrical invisibility cloak to electromagnetic waves, Physical Review B 76 (2007) 121101.

28. Y. Luo, J. Zhang, H. Chen, S. Xi, B.-I. Wu, Cylindrical cloak with axial permittivity/permeability spatially invariant, Applied Physics Letters 93 (2008) 033504.

29. W. Cai, U.K. Chettiar, A.V. Kildishev, V.M. Shalaev, G.W. Milton, Nonmagnetic cloak with minimized scattering, Applied Physics Letters 91 (2007) 111105. 
30. L. Huang, et al., Generalized transformation for nonmagnetic invisibility cloak with minimized scattering, JOSA B 28 (2011) 922-928.

31. B. Kanté, D. Germain, A. de Lustrac, Experimental demonstration of a nonmagnetic metamaterial cloak at microwave frequencies, Physical Review B 80 (2009) 201104.

32. W. Yan, M. Yan, M. Qiu, Non-magnetic simplified cylindrical cloak with suppressed zeroth order scattering, Applied Physics Letters 93 (2008) 021909.

33. J. Zhang, Y. Luo, N.A. Mortensen, Minimizing the scattering of a nonmagnetic cloak, Applied Physics Letters 96 (2010) 113511.

34. W. Cai, U.K. Chettiar, A.V. Kildishev, V.M. Shalaev, Optical cloaking with metamaterials, Nature Photonics 1 (2007) 224-227.

35. Z. Chang, X. Zhou, J. Hu, G. Hu, Design method for quasiisotropic transformation materials based on inverse Laplace's equation with sliding boundaries, Optics express 18 (2010) 6089-6096.

36. K. Yao, H. Chen, Y. Liu, X. Jiang, An analogy strategy for transformation optics, New Journal of Physics 16 (2014) 063008.

37. P. Zhang, M. Lobet, S. He, Carpet cloaking on a dielectric half-space, Optics Express 18 (2010) 18158-18163.

38. J. Li, J.B. Pendry, Hiding under the carpet: a new strategy for cloaking, Physical Review Letters 101 (2008) 203901.

39. P.A. Huidobro, M.L. Nesterov, L. Martin-Moreno, F.J. GarciaVidal, Transformation optics for plasmonics, Nano Letters 10 (2010) 1985-1990.

40. Y. Liu, T. Zentgraf, G. Bartal, X. Zhang, Transformational plasmon optics, Nano Letters 10 (2010) 1991-1997.

41. J. Renger, et al., Hidden progress: broadband plasmonic invisibility, Optics Express 18 (2010) 15757-15768.

42. R. Liu, et al., Broadband ground-plane cloak, Science 323 (2009) 366-369.

43. J. Valentine, J. Li, T. Zentgraf, G. Bartal, X. Zhang, An optical cloak made of dielectrics, Nature Materials 8 (2009) 568-571.

44. L.H. Gabrielli, J. Cardenas, C.B. Poitras, M. Lipson, Silicon nanostructure cloak operating at optical frequencies, Nature Photonics 3 (2009) 461-463.

45. F. Zhou, et al., Hiding a realistic object using a broadband terahertz invisibility cloak, Scientific Reports 1 (2011) 78.

46. D. Bao, et al., All-dielectric invisibility cloaks made of BaTiO3-loaded polyurethane foam, New Journal of Physics 13 (2011) 103023.

47. E. Kallos, C. Argyropoulos, Y. Hao, Ground-plane quasicloaking for free space, Physical Review A 79 (2009) 063825.

48. H.F. Ma, T.J. Cui, Compact-sized and broadband carpet cloak and free-space cloak, Optics Express 17 (2009) 19947-19959.

49. D. Shin, et al., Broadband electromagnetic cloaking with smart metamaterials, Nature Communications 3 (2012) 1213.

50. T. Ergin, J. Fischer, M. Wegener, Optical phase cloaking of $700 \mathrm{~nm}$ light waves in the far field by a three-dimensional carpet cloak, Physical Review Letters 107 (2011) 173901.

51. M. Gharghi, et al., A carpet cloak for visible light, Nano Letters 11 (2011) 2825-2828.

52. T. Ergin, N. Stenger, P. Brenner, J.B. Pendry, M. Wegener, Three-dimensional invisibility cloak at optical wavelengths, Science 328 (2010) 337-339.
53. H.F. Ma, T.J. Cui, Three-dimensional broadband ground-plane cloak made of metamaterials, Nature Communications 1 (2010) 21.

54. N.I. Landy, N. Kundtz, D.R. Smith, Designing three-dimensional transformation optical media using quasiconformal coordinate transformations, Physical Review Letters 105 (2010) 193902.

55. B. Zhang, T. Chan, B.-I. Wu, Lateral shift makes a groundplane cloak detectable, Physical Review Letters 104 (2010) 233903.

56. C. Li, X. Liu, F. Li, Experimental observation of invisibility to a broadband electromagnetic pulse by a cloak using transformation media based on inductor-capacitor networks, Physical Review B 81 (2010) 115133.

57. X. Liu, et al., Experimental verification of broadband invisibility using a cloak based on inductor-capacitor networks, Applied Physics Letters 95 (2009) 191107.

58. X. Sheng, C. Hongsheng, W. Bae-Ian, K. Jin Au, Onedirectional perfect cloak created with homogeneous material, IEEE Microwave and Wireless Components Letters 19 (2009) 131-133.

59. Y. Luo, J. Zhang, H. Chen, A rigorous analysis of planetransformed invisibility cloaks, IEEE Transactions on Antennas and Propagation 57 (2009) 3926-3933.

60. X. Chen, et al., Macroscopic invisibility cloaking of visible light, Nature Communications 2 (2011) 176.

61. B. Zhang, Y. Luo, X. Liu, G. Barbastathis, Macroscopic invisibility cloak for visible light, Physical Review Letters 106 (2011) 033901.

62. D. Liang, et al., Robust large dimension terahertz cloaking, Advanced Materials 24 (2012) 916-921.

63. X. Xu, Y. Feng, Y. Hao, J. Zhao, T. Jiang, Infrared carpet cloak designed with uniform silicon grating structure, Applied Physics Letters 95 (2009) 184102.

64. X. Xu, Y. Feng, Z. Yu, T. Jiang, J. Zhao, Simplified ground plane invisibility cloak by multilayer dielectrics, Optics Express 18 (2010) 24477-24485.

65. J. Zhang, L. Liu, Y. Luo, S. Zhang, N.A. Mortensen, Homogeneous optical cloak constructed with uniform layered structures, Optics Express 19 (2011) 8625-8631.

66. X. Xu, et al., Broad band invisibility cloak made of normal dielectric multilayer, Applied Physics Letters 99 (2011) 154104.

67. N. Landy, D.R. Smith, A full-parameter unidirectional metamaterial cloak for microwaves, Nature Materials 12 (2013) 25-28.

68. H. Chen, B. Zheng, Broadband polygonal invisibility cloak for visible light, Scientific Reports 2 (2012) 255.

69. T. Han, C. Qiu, X. Tang, An arbitrarily shaped cloak with nonsingular and homogeneous parameters designed using a twofold transformation, Journal of Optics 12 (2010) 095103.

70. W. Li, J. Guan, Z. Sun, W. Wang, Q. Zhang, A near-perfect invisibility cloak constructed with homogeneous materials, Optics Express 17 (2009) 23410-23416.

71. H. Chen, et al., Ray-optics cloaking devices for large objects in incoherent natural light, Nature Communications 4 (2013) 2652.

72. B. Edwards, A. Alù, M. Silveirinha, N. Engheta, Experimental verification of plasmonic cloaking at microwave frequencies with metamaterials, Physical Review Letters 103 (2009) 153901 . 
73. J.C. Soric, et al., Demonstration of an ultralow profile cloak for scattering suppression of a finite-length rod in free space, New Journal of Physics 15 (2013) 033037.

74. D. Rainwater, et al., Experimental verification of threedimensional plasmonic cloaking in free-space, New Journal of Physics 14 (2012) 013054.

75. A. Alù, Mantle cloak: Invisibility induced by a surface, Physical Review B 80 (2009) 245115.

76. M. Silveirinha, A. Alù, N. Engheta, Parallel-plate metamaterials for cloaking structures, Physical Review E 75 (2007) 036603.

77. Y. Ma, et al., First experimental demonstration of an isotropic electromagnetic cloak with strict conformal mapping, Scientific Reports 3 (2013) 2182.

78. S. Xu, et al., Experimental demonstration of a free-space cylindrical cloak without superluminal propagation, Physical Review Letters 109 (2012) 223903.

79. R. Schittny, M. Kadic, T. Buckmann, M. Wegener, Metamaterials Invisibility cloaking in a diffusive light scattering medium, Science 345 (2014) 427-429.

80. F. Gomory, et al., Experimental realization of a magnetic cloak, Science 335 (2012) 1466-1468.

81. S. Narayana, Y. Sato, DC magnetic cloak, Advanced Materials 24 (2012) 71-74.

82. J. Souc, et al., A quasistatic magnetic cloak, New Journal of Physics 15 (2013) 053019.

83. T. Han, et al., Manipulating DC currents with bilayer bulk natural materials, Advanced Materials 26 (2014) 3478-3483.

84. Q. Ma, Z.L. Mei, S.K. Zhu, T.Y. Jin, T.J. Cui, Experiments on active cloaking and illusion for laplace equation, Physical Review Letters 111 (2013) 173901.

85. F. Yang, Z.L. Mei, T.Y. Jin, T.J. Cui, DC electric invisibility cloak, Physical Review Letters 109 (2012) 053902.

86. F. Yang, et al., A negative conductivity material makes a dc invisibility cloak hide an object at a distance, Advanced Functional Materials 23 (2013) 4306-4310.

87. T. Mitchell, et al., Perfect surface wave cloaks, Physical Review Letters 111 (2013) 213901.

88. J.M. Lukens, D.E. Leaird, A.M. Weiner, A temporal cloak at telecommunication data rate, Nature 498 (2013) 205-208.

89. H. Chen, C.T. Chan, Acoustic cloaking in three dimensions using acoustic metamaterials, Applied Physics Letters 91 (2007) 183518 .
90. S. Cummer, et al., Scattering theory derivation of a $3 \mathrm{D}$ acoustic cloaking shell, Physical Review Letters 100 (2008) 024301 .

91. S.A. Cummer, D. Schurig, One path to acoustic cloaking, New Journal of Physics 9 (2007) 45-45.

92. A.N. Norris, Acoustic cloaking theory, Proceedings of the Royal Society A: Mathematical, Physical and Engineering Sciences 464 (2008) 2411-2434.

93. L. Sanchis, et al., Three-dimensional axisymmetric cloak based on the cancellation of acoustic scattering from a sphere, Physical Review Letters 110 (2013) 124301.

94. M. Farhat, S. Enoch, S. Guenneau, A. Movchan, Broadband cylindrical acoustic cloak for linear surface waves in a fluid, Physical Review Letters 101 (2008) 134501.

95. S. Zhang, C. Xia, N. Fang, Broadband acoustic cloak for ultrasound waves, Physical Review Letters 106 (2011) 024301.

96. B.-I. Popa, L. Zigoneanu, S.A. Cummer, Experimental acoustic ground cloak in air, Physical Review Letters 106 (2011) 253901.

97. L. Zigoneanu, B.I. Popa, S.A. Cummer, Three-dimensional broadband omnidirectional acoustic ground cloak, Nature Materials 13 (2014) 352-355.

98. T. Han, et al., Experimental demonstration of a bilayer thermal cloak, Physical Review Letters 112 (2014) 054302.

99. T. Han, T. Yuan, B. Li, C.W. Qiu, Homogeneous thermal cloak with constant conductivity and tunable heat localization, Scientific Reports 3 (2013) 1593.

100. Y. Ma, L. Lan, W. Jiang, F. Sun, S. He, A transient thermal cloak experimentally realized through a rescaled diffusion equation with anisotropic thermal diffusivity, NPG Asia Materials 5 (2013) e73.

101. H. Xu, X. Shi, F. Gao, H. Sun, B. Zhang, Ultrathin threedimensional thermal cloak, Physical Review Letters 112 (2014) 054301.

102. T. Buckmann, M. Thiel, M. Kadic, R. Schittny, M. Wegener, An elasto-mechanical unfeelability cloak made of pentamode metamaterials, Nature Communications 5 (2014) 4130.

103. M. Farhat, S. Guenneau, S. Enoch, , Ultrabroadband elastic cloaking in thin plates, Physical Review Letters 103 (2009) 024301 .

104. S. Zhang, D. Genov, C. Sun, X. Zhang, Cloaking of matter waves, Physical Review Letters 100 (2008) 123002.

Cite this article as: Yang Y, Zhang B, Li E \& Chen H: Towards omnidirectional, large scale, full polarization, and broadband practical invisibility cloaks: challenges and progress. EPJ Appl. Metamat. 2014, 1, 7. 